\title{
Alongamento estático versus conceito Mulligan: aplicações no treino de flexibilidade em ginastas
}

\author{
Static stretch versus Mulligan concept: flexibility training in gymnasts
}

\author{
Manuela Karloh ${ }^{[\mathrm{a}]}$, Raquel Petry dos Santos ${ }^{[\mathrm{b}]}$, Maria Helena Kraeski ${ }^{[\mathrm{c}]}$, Thiago Sousa Matias ${ }^{[\mathrm{d}]}$, \\ Daniel Kraeski ${ }^{[\mathrm{e}]}$, Fabio Sprada de Menezes ${ }^{[\mathrm{f}]}$
}

[a] Mestranda em Ciências do Movimento Humano, Centro de Ciências da Saúde e do Esporte, Universidade do Estado de Santa Catarina (CEFID/UDESC), Florianópolis, SC - Brasil, e-mail: manukarloh@gmail.com

[b] Fisioterapeuta da equipe de ginástica rítmica da Universidade do Estado de Santa Catarina (ADIEE/UDESC), Florianópolis, SC - Brasil, e-mail: raqpetry@yahoo.com.br

[c] Coordenadora técnica da equipe de ginástica rítmica da Universidade do Estado de Santa Catarina (ADIEE/UDESC), Florianópolis, SC - Brasil, e-mail: gr_adiee@yahoo.com.br

[d] Mestrando em Ciências do Movimento Humano, Centro de Ciências da Saúde e do Esporte, Universidade do Estado de Santa Catarina (CEFID/UDESC), Florianópolis, SC - Brasil, e-mail: thiagosousamatias@gmail.com

[e] Preparador Físico da equipe de ginástica rítmica da Universidade do Estado de Santa Catarina (ADIEE/UDESC), Florianópolis, SC - Brasil, e-mail: dkraeski@hotmail.com

[f] Mestre em Ciências do Movimento Humano, Centro de Ciências da Saúde e do Esporte, Universidade do Estado de Santa Catarina (CEFID/UDESC), Florianópolis, SC - Brasil, e-mail: fabio_1711@yahoo.com.br

\section{Resumo}

Objetivos: Investigar e comparar as adaptações agudas do treino de flexibilidade da articulação coxofemoral no movimento de extensão em atletas de ginástica rítmica, em função de duas técnicas de alongamento: o alongamento estático e a técnica Mulligan - Long Leg Traction. Metodologia: Este é um estudo de caso, de natureza quase-experimental. Participaram do estudo oito atletas do sexo feminino com média de idade de 13,25 anos, divididas em dois grupos. O Grupo 1 foi submetido à técnica Mulligan - Long Leg Traction e o Grupo 2 ao alongamento estático. Utilizou-se a fotogrametria para avaliar a amplitude de movimento de extensão da articulação coxofemoral. Realizou-se a avaliação da amplitude de movimento antes do início do período de treinamento, antes e imediatamente após cada sessão. O protocolo teve duração de seis semanas (11 sessões de treinamento). Utilizou-se estatística descritiva e inferencial para o tratamento dos dados. Resultados: A amplitude de movimento inicial de extensão do quadril foi $31,38^{\circ}$ no membro inferior esquerdo e $30,35^{\circ}$ no membro inferior direito. Quanto às adaptações agudas ao 
alongamento, o ganho do Grupo 1 (4,73 \pm 0,62 e 4,92 \pm 0,26 $6^{\circ}$ no membro inferior esquerdo e direito respectivamente) foi estatisticamente superior de modo significativo ao do Grupo 2 (3,42 \pm 0,58 no membro inferior esquerdo e $3,5 \pm 0,70^{\circ}$ no membro inferior direto). Conclusões: Com relação às adaptações agudas, o Grupo 1 (Mulligan) obteve ganhos estatisticamente significativos superiores que o Grupo 2 (alongamento estático).

Palavras-chave: Flexibilidade. Exercícios de alongamento muscular. Mulligan. Ginástica rítmica.

\begin{abstract}
Purpose: To investigate and compare the acute changes in flexibility of hip extension in athletes of Rhythmic Gymnastics in terms of two stretching techniques: the static stretch and Mulligan's Long Leg Traction. Methods: this is a case study, with a quasi experimental investigation. Participated eight female athletes with an average age of 13.25 years, divided into two groups. Group 1 performed Mulligan's Long Leg Traction and Group 2 performed static stretch. Photogrammetry was used to assess the range of motion of hip extension. The range of motion was assessed before the program beginning, before and immediately after each session. The duration of the protocol was six weeks (11 sessions). Data were analyzed using descriptive and inferential statistics. Results: Initial range of motion of hip extension was $31.38^{\circ}$ in the left lower limb and $30.35^{\circ}$ in right lower limb. Regarding the acute effects of stretching, the gain in Group $1\left(4.73 \pm 0.62^{\circ}\right.$ and $4.92 \pm 0.26^{\circ}$ in the left and right lower limb, respectively) was statistically significant superior than in Group $2\left(3.42 \pm 0.58^{\circ}\right.$ in left lower limb and $3.5 \pm 0.70^{\circ}$ in right lower limb). Conclusions: With respect to acute changes, Group 1 (Mulligan) had statistically greater gains than Group 2 (static stretching).
\end{abstract}

Keywords: Flexibility. Muscle stretching exercises. Mulligan. Rhythmic gymnastics.

\title{
Introdução
}

A ginástica rítmica (GR) é um esporte que se destaca pela elegância e beleza dos movimentos e requer alto nível de desenvolvimento de algumas qualidades físicas, objetivando a perfeição técnica na execução dos elementos corporais e no manejo dos aparelhos (1).

A flexibilidade tem um papel decisivo nas modalidades esportivas cujo componente estético é marcante, uma vez que exigem a utilização completa dos arcos articulares, pois determinados gestos não poderiam ser realizados adequadamente na ausência de amplitudes de movimento elevadas (2). Dessa forma, na GR a flexibilidade é uma das valências físicas mais importantes, além de ser um elemento corporal previsto pelo Código de Pontuação da GR da Federação Internacional de Ginástica. Os gestos esportivos deste esporte têm como característica a utilização de grandes amplitudes de movimento das articulações, principalmente dos movimentos de extensão da coluna vertebral e da articulação coxofemoral (1).

O aprimoramento da flexibilidade desenvolve-se pela execução de alongamentos. Três técnicas de alongamento são comumente sugeridas para o ganho de flexibilidade no esporte: alongamento dinâmico ou balístico, alongamento estático ou passivo e a Facilitação Neuromuscular Proprioceptiva - FNP (3, 4).

O alongamento estático é o método mais comum para o ganho de flexibilidade e oferece vantagens quando comparado às outras duas formas de alongamento, graças à facilidade de execução e ao baixo potencial de dano tecidual (4-6).

Além desses métodos já descritos, encontram-se evidências na literatura da utilização de técnicas provenientes do conceito Mulligan para o aprimoramento da flexibilidade (7). Tais técnicas consistem na associação de alongamentos estáticos com trações e têm por objetivo aumentar a amplitude de movimento e aprimorar a flexibilidade de flexão, extensão, adução e abdução do quadril $(8,9)$. Estudos demonstram a aplicação destas técnicas em grupos musculares localizados nos membro inferiores, tais como os isquiotibiais 
e os adutores do quadril, com ganhos similares ou superiores aos das técnicas de alongamentos convencionais $(7,9)$. Estas evidências confirmam a hipótese de que técnicas do Conceito Mulligan podem gerar uma amplitude de movimento maior que outras técnicas convencionais.

Levando em consideração o descrito e o fato de não se conhecerem estudos que utilizam o conceito Mulligan no ganho de amplitude de movimento em ginastas, este estudo tem como objetivo avaliar a amplitude de movimento de extensão da articulação coxofemoral, além de comparar as adaptações agudas do treino desta em atletas de ginástica rítmica, em função de duas técnicas de alongamento: o alongamento estático e a técnica Mulligan - Long Leg Traction.

\section{Materiais e método}

Este é um estudo de caso de natureza quase-experimental e caracteriza-se por uma abordagem quantitativa (10). O estudo foi aprovado pelo Comitê de Ética em Pesquisa em Seres Humanos da Universidade do Estado de Santa Catarina - CEP/UDESC, sob o número 88/2008.

\section{Participantes}

Participam do estudo oito atletas de ginástica rítmica com idades entre 12 e 16 anos, integrantes da equipe ADIEE/UDESC de Florianópolis, SC. A média de idade das atletas participantes foi de 13,25 \pm 0,89 anos.

A escolha dos sujeitos foi realizada intencionalmente e por conveniência. Os critérios de inclusão foram: praticantes de GR, com idade entre 12 e 16 anos. Determinou-se como critério de exclusão lesões músculo-esqueléticas agudas ou crônicas que influenciem, comprometam ou limitem a amplitude de movimento da articulação coxofemoral.

\section{Instrumentos da avaliação e coleta dos dados}

Para avaliar a amplitude de movimento (ADM) de extensão da articulação do quadril utilizou-se a fotogrametria. Os ângulos articulares foram analisados e quantificados pelo software AutoCAD ${ }^{\circledR} 2007$. Para o registro fotográfico foi utilizada uma máquina fotográfica digital, Sony ${ }^{\circledR}$, modelo DSC T5, 5.1 megapixels de resolução, lente Carl Zeiss Vario-Tessar fixada sobre um tripé Vivitar ${ }^{\mathbb{B}}$ de 1,30 metros de altura. Utilizou-se a distância de 3 metros entre o local no qual as atletas foram posicionadas e o tripé, a fim de que a divergência produzida pela lente convencional da câmera não interferisse nas mensurações dos ângulos articulares de modo significativo (11). Utilizou-se também uma cinta Mulligan ${ }^{\circledR}$ para auxiliar a aplicação de uma das técnicas propostas.

\section{Avaliação inicial}

A avaliação inicial foi realizada antes de iniciar os treinos de flexibilidade, e compreendeu a anamnese (contendo as iniciais do nome da atleta, idade, tempo de prática de GR, membro inferior dominante e a ocorrência de lesões músculo-esqueléticas prévias nos membros inferiores) e a avaliação da amplitude de movimento de extensão do quadril. Para tal, as atletas foram posicionadas sobre um cavalo de saltos oficial da ginástica artística. Realizou-se também a marcação dos pontos de referência articular: epicôndilo lateral do fêmur, trocânter maior do fêmur e lóbulo da orelha (12).

O ângulo mensurado correspondeu ao movimento de extensão do quadril e constitui-se da intersecção da reta formada pelos pontos epicôndilo lateral do fêmur e trocânter maior do fêmur, com a reta que parte do lóbulo da orelha, traçada paralelamente à superfície do local onde foi posicionada a atleta. Para a mensuração 
utilizou-se a posição do Teste de Thomas modificado (13). O movimento de extensão do quadril foi executado passivamente pelo avaliador até o ponto em que a atleta conseguisse manter a coluna lombar retificada e em contato com a superfície do cavalo de saltos. A articulação do joelho foi mantida livre. O registro fotográfico deu-se na posição de máxima amplitude de movimento da extensão do quadril.

\section{Treinos de flexibilidade - intervenção nos Grupos 1 e 2}

Para a aplicação dos protocolos propostos, as atletas foram divididas por ranqueamento de acordo com a amplitude de movimento de extensão do quadril encontrada na avaliação inicial. Sendo assim, as atletas receberam uma classificação de 1 a 8: o valor 1 corresponde ao melhor resultado no teste de flexibilidade e o valor 8 ao pior. A atleta número 1 será indicada ao primeiro grupo, número 2 ao segundo, a 3 ao primeiro, a 4 ao segundo e assim sucessivamente. Este procedimento permitirá que os grupos fiquem homogêneos e semelhantes (10).

A designação do método de treinamento dos Grupos 1 e 2 foi realizada por sorteio. O grupo 1 foi composto por quatro atletas submetidas ao treino de flexibilidade utilizando apenas a técnica Mulligan Long Leg Traction (alongamento estático associado à tração do segmento) para extensão do quadril (Figura 1). O Grupo 2 foi composto por quatro atletas que foram submetidas ao treino de flexibilidade utilizando somente o alongamento estático (Figura 2).

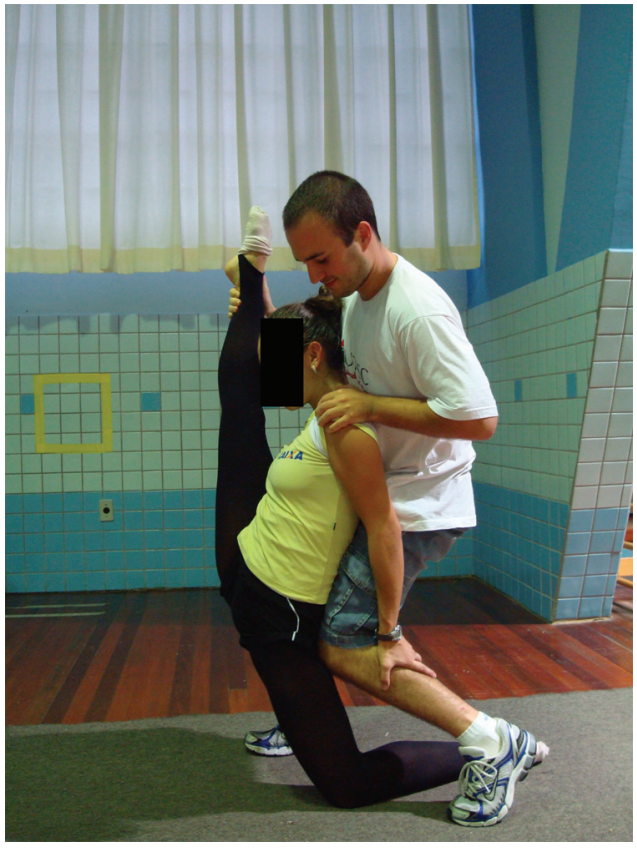

Figura 1 - Mulligan Long Leg Traction

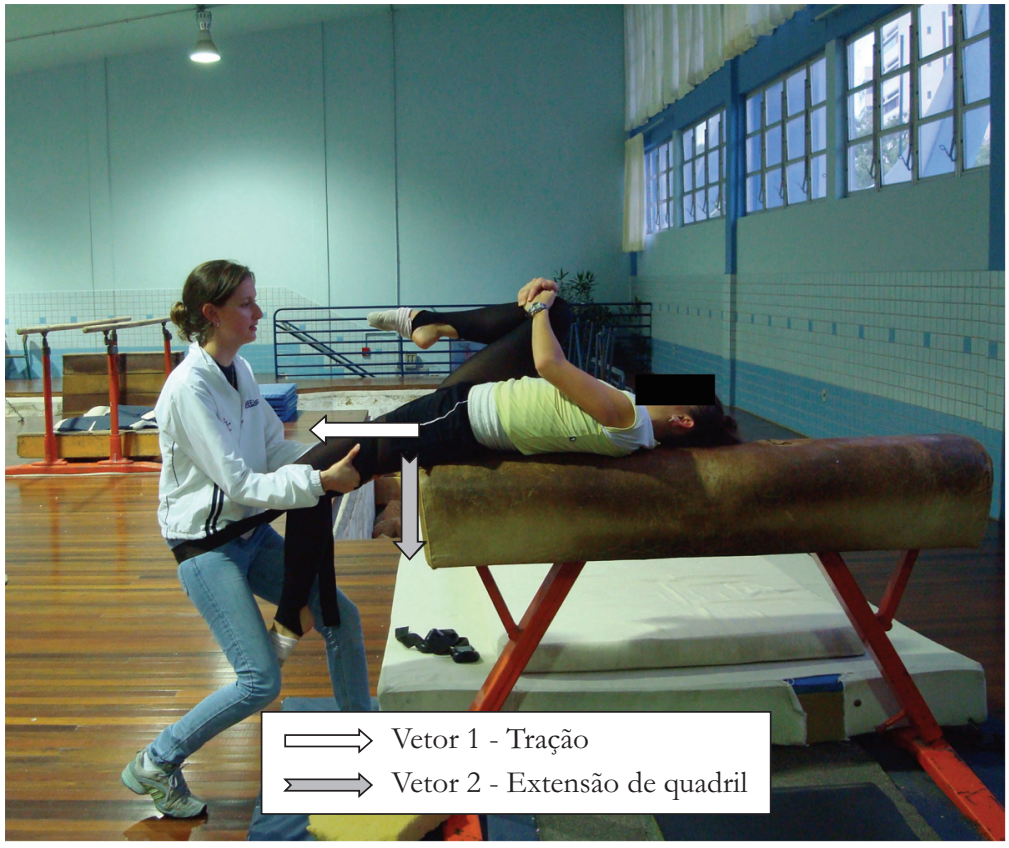

Figura 2 - Alongamento estático

Antes e imediatamente após a aplicação de cada treino, as atletas de ambos os grupos foram submetidas à mensuração da ADM de extensão da articulação coxofemoral, esta realizada de forma idêntica à avaliação inicial.

As coletas de dados foram realizadas no período de 15 de julho a 15 de setembro de 2008 no ginásio onde são realizados os treinamentos da equipe. Neste período realizaram-se 11 sessões de treinos de flexibilidade para cada um dos grupos. Os treinos de flexibilidade aconteceram duas vezes na semana, sempre após o aquecimento das atletas, no qual se executou uma rotina básica de exercícios com cerca de 50 minutos de duração. Em cada dia de treinamento realizaram-se duas repetições da intervenção proposta (Mulligan ou 
alongamento estático) em cada membro inferior, com duração de 30 segundos e repouso de 30 segundos entre as séries $(5,14,15)$.

\section{Tratamento dos dados}

Os dados foram sistematizados no programa SPSS ${ }^{\circledR}$ versão 16.0. Para a análise dos resultados, utilizou-se estatística descritiva - mínimo, máximo, média e desvio padrão. Previamente aos testes inferenciais, realizou-se uma análise exploratória dos dados, verificaram-se os valores de assimetria e curtose, bem como a normalidade com o teste de Shapiro-Wilk. Como a distribuição dos dados foi não paramétrica, foram utilizados o teste de Wilcoxon para o delineamento pré e pós-teste intragrupo e o teste de Mann-Whitney para a comparação intergrupos. A significância foi de $\mathrm{p}<0,05$.

\section{Resultados e discussão}

\section{Avaliação inicial}

A amplitude de movimento inicial do movimento de extensão da articulação coxofemoral dos sujeitos do estudo variou entre $25^{\circ}$ e $39^{\circ}$ no membro inferior esquerdo (MIE), sendo a média $31,38 \pm 5,26^{\circ}$. No membro inferior direito (MID) o valor mínimo foi $25^{\circ}$ e o máximo $38^{\circ}$, com média de $30,35 \pm 4,04^{\circ}$.

Steinberg et al. (16) analisaram a amplitude de movimento de extensão do quadril em bailarinos e não bailarinos de diversas faixas etárias. Nos bailarinos de 12 a 16 anos, a amplitude de movimento encontrada variou de 30 a 35, valores similares aos encontrados no nosso estudo. Enquanto para os não bailarinos, os valores foram próximos a $20^{\circ}$.

Observou-se com isso que a flexibilidade é uma característica marcante nos esportes cujo componente estético está presente, dentre os quais estão a GR. Já para outras modalidades esportivas e para indivíduos não atletas a flexibilidade desta articulação não é uma característica marcante. O estudo de L'Hermette et al. (17) analisou a flexibilidade do quadril de 97 atletas de handebol, sendo que estas apresentaram em média $6^{\circ}$ de extensão de quadril.

\section{Adaptações agudas ao alongamento}

\section{Adaptações agudas ao alongamento no Grupo 1 (Grupo Mulligan)}

A média da amplitude do movimento de extensão da articulação coxofemoral dos sujeitos submetidos ao alongamento Long Leg Traction, mensurada antes de cada uma das 11 sessões de treinamento, foi de 36,04 ${ }^{\circ}$

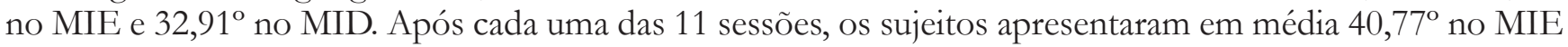
e $37,79^{\circ}$ no MID (ganho de 4,73 \pm 0,62 e 4,92 $\pm 0,26^{\circ}$ na ADM do MIE e MID, respectivamente). Apesar do aumento clínico na amplitude de movimento de ambos os membros inferiores, este não foi considerado significativo em grande parte dos 11 treinamentos (Gráfico 1).

Alguns estudos têm demonstrado a eficiência das técnicas de Mulligan no ganho de amplitude de movimento: Hall et al. (7) demonstraram um aumento de $13,3^{\circ}$ na flexão de quadril após a aplicação de três repetições da mesma técnica utilizada no presente estudo. Mais recentemente, os mesmos autores descrevem um aumento de $11^{\circ}$ na flexão do quadril imediatamente após a intervenção (9). Meszaros et al. (18) também observaram o aumento na amplitude de movimento dos membros inferiores após a aplicação desta técnica. Deve-se considerar que os ganhos encontrados na literatura são superiores aos do presente estudo, visto que nestes casos a população investigada foi de indivíduos não atletas, além de representarem a amplitude de movimento de flexão de quadril, que fisiologicamente atinge valores superiores aos de extensão do quadril (19). 


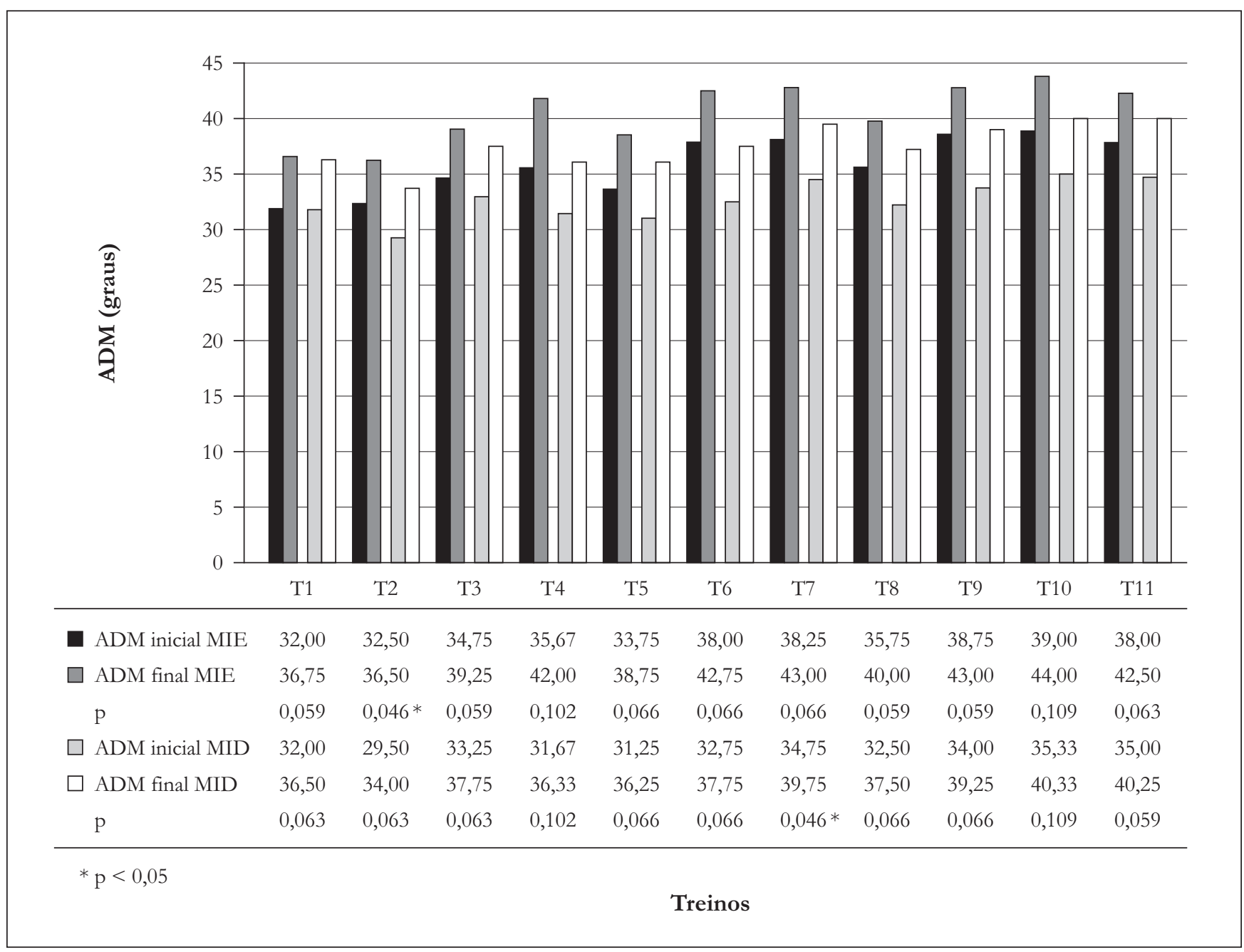

Gráfico 1 - Adaptações agudas ao alongamento no Grupo 1 (Grupo Mulligan) - Amplitudes de movimento inicial e final em cada uma das sessões de treinamento

\section{Adaptações agudas ao alongamento no Grupo 2 (Grupo alongamento estático)}

A média da amplitude de movimento do movimento de extensão da articulação coxofemoral dos sujeitos submetidos ao alongamento estático, mensurada antes de cada uma das 11 sessões de treinamento, foi de $36,73^{\circ}$ no MIE e 32,64 no MID. Após cada uma das 11 sessões, os sujeitos apresentaram em média $40,15^{\circ}$ no MIE e $36,13^{\circ}$ no MID (ganho de 3,42 $\pm 0,58^{\circ}$ no MIE e 3,5 $\pm 0,70^{\circ}$ no MID).

Apesar do aumento clínico na amplitude de movimento de ambos os membros inferiores, este não foi considerado significativo na maioria dos 11 treinamentos (Gráfico 2).

O estudo de Whatman, Knappsteina e Hume (20) demonstrou ganhos imediatos de $4^{\circ}$ a $5^{\circ}$. Tais valores são similares aos observados no presente estudo.

A literatura tem demonstrado a eficiência dos alongamentos estáticos no ganho de amplitude de movimento de diversas articulações. Halbertsma, Van Bolhuis e Goeken (21) demonstraram aumento de 8,9 da flexão de quadril imediatamente após a aplicação do alongamento estático. Feland et al. (22) observaram aumento de $1,3^{\circ}$ por semana, num total de seis semanas, nos escores de flexibilidade dos isquiotibiais após a intervenção com a mesma técnica de alongamento. Zakas et al. (15) reportaram o incremento significativo na 


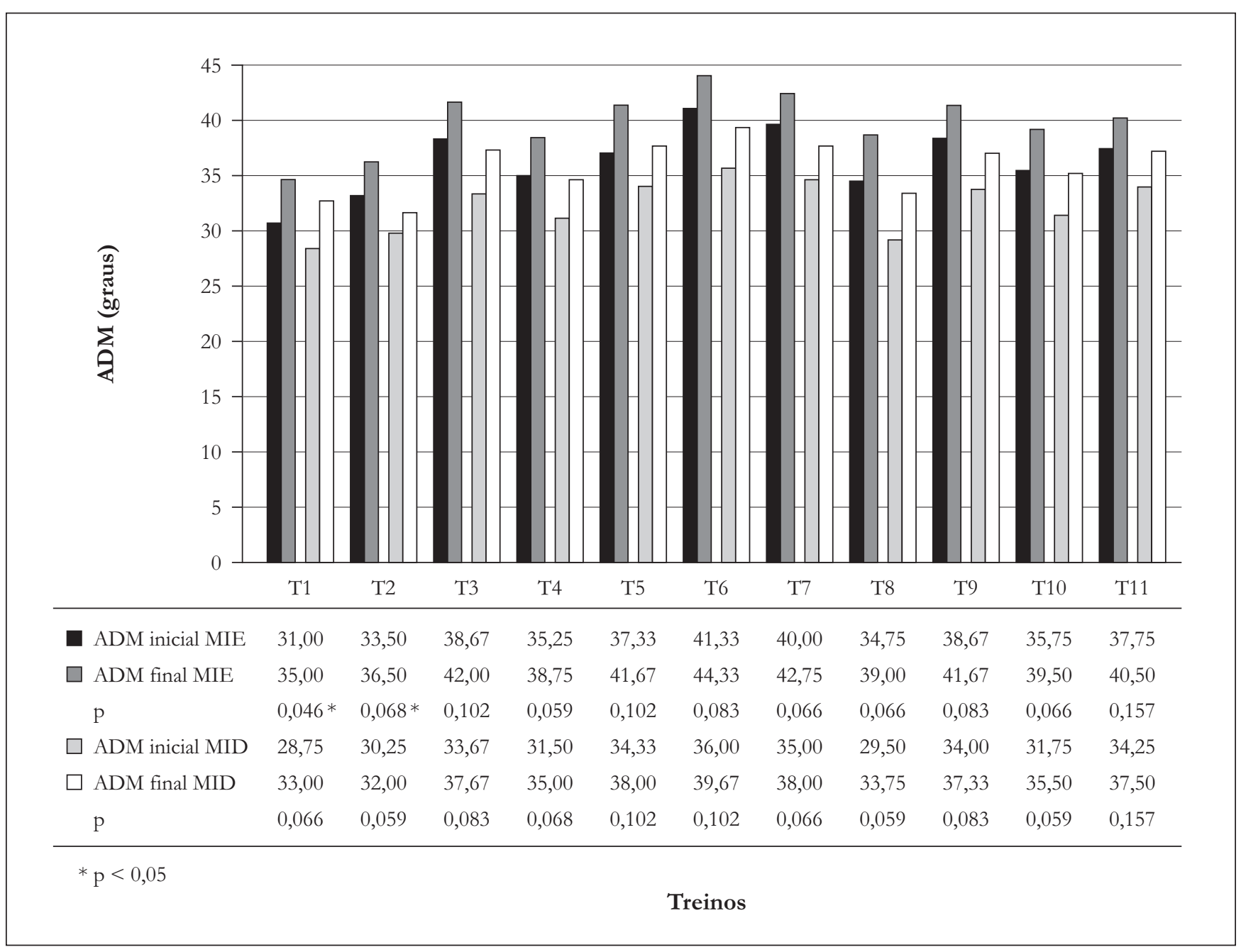

Gráfico 2 - Adaptações agudas ao alongamento no Grupo 2 (Grupo Alongamento Estático) - Amplitudes de movimento inicial e final em cada uma das sessões de treinamento

amplitude de movimento de flexão $\left(9,4^{\circ}\right)$, extensão $\left(6,3^{\circ}\right)$ e abdução $\left(4,9^{\circ}\right)$ de quadril, flexão de joelho $\left(4,2^{\circ}\right)$, dorsiflexão do tornozelo $\left(2,8^{\circ}\right)$ após a execução de alongamentos estáticos (uma série de 30 segundos).

Pode-se observar que os ganhos relatados na literatura são superiores aos observados nas ginastas. Provavelmente este achado se deve ao fato de a ginástica rítmica ser um esporte que naturalmente exige grandes amplitudes de movimento, principalmente do quadril. Estas atletas já trabalham com amplitudes de movimento extremas, não sendo observadas, assim, grandes variações destas. Entretanto, estes achados não invalidam o trabalho de flexibilidade com as ginastas, uma vez que contribui para a execução dos movimentos exigidos por este esporte.

\section{Adaptações agudas ao alongamento - Grupo 1 versus Grupo 2}

Observou-se ganho imediato de amplitude de movimento nos dois grupos investigados. Entretanto, o aumento da amplitude de movimento imediatamente após os treinos de flexibilidade foi estatisticamente superior de modo significativo no Grupo 1 (Grupo Mulligan) (MIE e MID p = 0,000) (Tabela 1). Este 
apresentou o aumento de 4,73 $\pm 0,62^{\circ}$ e 4,92 $\pm 0,26^{\circ}$ na $\mathrm{ADM}$ do MIE e MID respectivamente; enquanto que o Grupo 2 apresentou um aumento de 3,42 $\pm 0,58^{\circ}$ no MIE e 3,5 $\pm 0,70^{\circ}$ no MID.

O conhecimento dos efeitos agudos dos alongamentos é importante para determinar a eficiência de um dado método de treinamento e para adquirir o aumento na amplitude de movimento antes de uma determinada atividade (22). Três mecanismos têm sido descritos na literatura como responsáveis pelo aumento da amplitude de movimento imediatamente após os exercícios de alongamento:

1) acredita-se que ocorra um aumento da tolerância ao alongamento. Tal fenômeno envolve alterações na percepção do alongamento por meio da modificação no limiar de sensibilidade dos receptores de dor $(5,6,23-25)$;

2) o mecanismo neurofisiológico é explicado pela inibição reflexa do músculo submetido ao alongamento. Essa resposta reflexa diminui a atividade dos componentes contráteis do músculo e resulta no aumento de sua extensibilidade e, consequentemente, um aumento na amplitude de movimento (20);

3) o terceiro mecanismo é baseado nas propriedades viscoelásticas do tecido. Os tecidos biológicos submetidos à tensão apresentam uma resposta viscoelástica, o que permite o alongamento tecidual. Baseados nessa propriedade, alguns autores sugerem que ocorra a diminuição na tensão e rigidez da unidade músculo-tendínea imediatamente após o alongamento passivo (14, 20, 25-27).

A diminuição na tensão e rigidez da unidade músculo-tendínea imediatamente após o alongamento parece ser um mecanismo eficaz em atividades que requerem extremas amplitudes de movimento (20). No entanto, o aumento na extensibilidade do tecido conectivo reduz a sensibilidade dos fusos neuromusculares, reduzindo possivelmente a velocidade da ativação muscular e, consequentemente, a capacidade de geração de força após os exercícios de alongamento (28).

Portanto, em termos pragmáticos, os exercícios de alongamento antes de atividades esportivas, particularmente naquelas que requerem altos níveis de flexibilidade, como a ginástica e a dança, podem ser necessários para alcançar máxima amplitude de movimento e possibilitar o aumento na performance (20, 28).

Entretanto, deve-se buscar um equilíbrio na aplicação de treinos de flexibilidade antes de períodos competitivos, para que sejam assegurados níveis elevados de flexibilidade sem comprometer a geração de força (28).

Tabela 1 - Adaptações agudas ao alongamento - ganhos observados após cada sessão de treinamento nos grupos 1 e 2

\begin{tabular}{|c|c|c|c|c|c|c|c|c|c|c|c|c|c|}
\hline & $\mathrm{T} 1$ & T2 & T3 & T4 & T5 & T6 & T7 & T8 & T9 & T10 & T11 & Média \pm DP & $\mathrm{p}$ \\
\hline $\begin{array}{l}\text { G1 } \\
\text { MIE }\end{array}$ & $4,75^{\circ}$ & $4,00^{\circ}$ & $4,50^{\circ}$ & $6,33^{\circ}$ & $5,00^{\circ}$ & $4,75^{\circ}$ & $4,75^{\circ}$ & $4,25^{\circ}$ & $4,25^{\circ}$ & $5,00^{\circ}$ & $4,50^{\circ}$ & $4,73 \pm 0,62^{\circ}$ & $0,000^{*}$ \\
\hline $\begin{array}{l}\text { G2 } \\
\text { MIE }\end{array}$ & $4,00^{\circ}$ & $3,00^{\circ}$ & $4,00^{\circ}$ & $3,5^{\circ}$ & $4,33^{\circ}$ & $3,00^{\circ}$ & $2,75^{\circ}$ & $4,25^{\circ}$ & $3^{\circ}$ & $3,75^{\circ}$ & $2,75^{\circ}$ & $4,92 \pm 0,26^{\circ}$ & \\
\hline $\begin{array}{l}\text { G1 } \\
\text { MID }\end{array}$ & $5,00^{\circ}$ & $4,50^{\circ}$ & $4,50^{\circ}$ & $4,67^{\circ}$ & $5,00^{\circ}$ & $5,00^{\circ}$ & $5,00^{\circ}$ & $5,00^{\circ}$ & $5,25^{\circ}$ & $5,00^{\circ}$ & $5,25^{\circ}$ & $3,42 \pm 0,58^{\circ}$ & $000 *$ \\
\hline $\begin{array}{l}\text { G2 } \\
\text { MID }\end{array}$ & $4,25^{\circ}$ & $1,75^{\circ}$ & $4,00^{\circ}$ & $3,50^{\circ}$ & $3,67^{\circ}$ & $3,67^{\circ}$ & $3,00^{\circ}$ & $4,25^{\circ}$ & $3,33^{\circ}$ & $3,75^{\circ}$ & $3,25^{\circ}$ & $3,50 \pm 0,70^{\circ}$ & \\
\hline
\end{tabular}

G1MIE: Ganho do membro inferior esquerdo do grupo 1 (Mulligan); G2MIE: Ganho do membro inferior esquerdo do grupo 2 (Alongamento Estático); G1MID: Ganho do membro inferior direito do grupo 1 (Mulligan); G2MID: Ganho do membro inferior direito do grupo 2 (Alongamento Estático); T1: treino 1; T2: treino 2; T3: treino 3; T4: treino 4; T5: treino 5; T6: treino 6; T7: treino 7; T8: treino 8; T9: treino 9; T10: treino 10; T11: treino $11 .{ }^{*} \mathrm{p}<0,05$. 
Em relação ao maior ganho imediato de flexibilidade no Grupo 1, a literatura descreve que este tipo de técnica (Mulligan - Long Leg Traction) é mais eficaz no aumento de amplitude de movimento graças à associação da tração ao alongamento estático. Além dos efeitos promovidos pelo alongamento estático, a tração do membro provavelmente inicia várias vias de reflexos no sistema nervoso central, incluindo aquelas mediadas pelo órgão tendinoso de Golgi aferente 1b, que conduz impulso excitatório do interneurônio 1b; e também aquelas conduzidas pelo fuso muscular tipo 2 aferentes. Os interneurônios $1 \mathrm{~b}$ inibem o neurônio motor alfa da musculatura alongada e excitam o neurônio motor alfa da musculatura antagonista. O órgão tendinoso de Golgi, sendo relativamente insensível a alongamentos vagarosamente aplicados, é menos propenso a ter resposta pelo efeito inibitório do que o fuso muscular tipo 2 aferente. O interneurônio $1 \mathrm{~b}$ aferente também recebe estímulo significativo dos aferentes 1a e aferentes articulares. Uma inibição adicional é alcançada por meio de vias supraespinais como os tratos córtico-espinal, rubro-espinal e retículo-espinal. Estes mecanismos podem, então, inibir a ação dos músculos alongados, permitindo maior amplitude de movimento, sendo esta geralmente livre de dor (7).

\section{Limitações do Estudo}

O presente estudo restringiu-se em analisar somente os efeitos agudos promovidos pelos exercícios de alongamento. Porém, um estudo prévio aplicado na mesma população estudada demonstrou as adaptações crônicas promovidas pelos exercícios de alongamento após um período de seis semanas de treinamento (28).

Apesar do contributo que se possa retirar deste estudo, é necessário ter em consideração que este estudo possui algumas limitações. Trata-se de um estudo de casos com ginastas de uma única equipe; assim, não deve ser considerada representativa de toda a população de ginastas. Além disso, o número reduzido da amostra pode ter interferido no poder estatístico do estudo. Seria também interessante levar em consideração o controle das variáveis que possam influenciar o estudo e, ainda, um delineamento metodológico de natureza longitudinal, no sentido de avaliar a resposta da flexibilidade ao longo das diferentes fases e tipos de treinamento.

\section{Considerações finais}

No que se refere às adaptações agudas ao alongamento, foram observados incrementos na amplitude de movimento de extensão da articulação coxofemoral após cada uma das 11 sessões de treinamento em ambos os grupos; porém o Grupo 1 (Mulligan) obteve ganhos estatisticamente superiores aos do Grupo 2 (alongamento estático). Esses resultados podem auxiliar a prescrição de exercícios de alongamento, tanto para os períodos de treinamento quanto para os períodos competitivos da equipe em questão. Por se tratar de um estudo de caso, este não deve ser considerado representativo de toda a população de ginastas. Sugere-se a realização de estudos semelhantes em um grupo maior de atletas de ginástica rítmica e de outras modalidades esportivas.

\section{Referências}

1. Hutchinson MR. Low back pain in elite rhythmic gymnasts. Med Sci Sports Exerc. 1999;31(11):1686-8.

2. Dupont P. Medical follow-up and lumbopelvic flexibility in rhythmic gymnastics. J Traumatol Sport. 2005;22(4):213-8.

3. Funk DC, Swank AM, Mikla BM, Fagan TA, Farr BK. Impact of prior exercise on hamstring flexibility: a comparison of proprioceptive neuromuscular facilitation and static stretching. J Strength Cond Res. 2003;17(3):489-2.

4. Roberts JM, Wilson K. Effect of stretching duration on active and passive range of motion in the lower extremity. Br J Sports Med. 1999;33(4):259-63.

5. Bandy WD, Irion JM. The effect of time on static stretch on the flexibility of the hamstring muscles. Phys Ther. 1994;74(9):845-50. 
6. Bandy WD, Irion JM, Briggler M. The effect of time and frequency of static stretching on flexibility of the hamstring muscles. Phys Ther. 1997;77(10):1090-6.

7. Hall TM, Cacho A, McNee C, Riches J, Walsh J. Effects of the Mulligan traction straight leg raise technique on range of movement. J Man Manipulative Ther. 2001;9(3):128-33.

8. Mulligan BR. Manual therapy 'NAGS', 'SNAGS', 'MWMS' etc. 4th ed. Wellington: Hutcheson Bowman \& Stewart; 1999.

9. Hall TM, Beyerlein G, Hansson U, Um HT, Odermark M, Sainsbury D. Mulligan traction straight leg raise: a pilot study to investigate effects on range of motion in patients with low back pain. J Man Manipulative Ther. 2006;14(2):95-100.

10. Thomas JR, Nelson JK. Métodos de pesquisa em atividade física. 3a ed. Porto Alegre: Artmed; 2002.

11. Braz RG, Goes FPDC, Carvalho GA. Confiabilidade e validade de medidas angulares por meio do software para avaliação postural. Fisioter Mov. 2008;21(3):117-26.

12. Dantas EHM, CarvalhoJLT, Fonseca RMO. Protocolo LABIFIE de goniometria. Rev Trein Desp. 1997;2(3):21-34.

13. Harvey D. Assessment of the flexibility of elite athletes using the modified Thomas test. Br J Sports Med. 1998;32(1):68-70.

14. Taylor DC, Dalton JD, Seaber AV, Garrett WE. Viscoelastic properties of muscle-tendon units: the biomechanical effects of stretching. Am J Sports Med. 1990;18(3):300-9.

15. Zakas A. The effect of stretching duration on the lower extremity flexibility of adolescent soccer players. J Bod Mov Ther. 2005;9(3):220-5.

16. Steinberg N, Hershkovitz I, Peleg S, Dar G, Masharawi Y, Heim M, et al. Range of joint movement in female dancers and non dancers aged 8 to 16 years: anatomical and clinical implication. Am J Sports Med. 2006;34(5):814-23.

17. L'Hermette M, Polle G, Toumv-Chollet, Duiardin F. Hip passive range of motion and frequency of radiographic hip osteoarthritis in former elite handball players. Br J Sports Med. 2006;40(1):45-9.

18. Meszaros TF, Olson R, Kulig K, Creighton D, Czarnecki E. Effect of 10\%,30\%, and $60 \%$ body weight traction on the straight leg raise test of symptomatic patients with low back pain. J Orthop Sports Phys Ther. 2000;30(10):595-601.

19. Mccreary EK, Provance PG, Kendall FP, Rodgers M, Romani WA. Músculos: provas e funções. 5a ed. São Paulo: Manole; 2007.

20. Whatman C, Knappsteina A, Hume P. Acute changes in passive stiffness and range of motion post-stretching. Physical Therapy in Sport. 2006;7(4):195-200.

21. Halbertsma JPK, Van Bolhuis AI, Goeken LNH. Sport stretching: effect on passive muscle stiffness of short hamstrings. Arch Phys Med Rehabil. 1996;77(7):688-92.

22. Feland JB, Myrer JW, Schulthies SS, Fellingham GW, Measom GW. The effect of duration of stretching of the hamstring muscle group for increasing range of motion in people aged 65 years or older. Phys Ther. 2001;81(5):1110-7.

23. Halbertsma JP, Goeken LN. Stretching exercises: effect on passive extensibility and stiffness in short hamstrings of healthy subjects. Arch Phys Med Rehabil. 1994;75(9):976-81.

24. Halbertsma JP, Mulder I, Göeken LN, Eisma WH. Repeated passive stretching: acute effect on the passive muscle moment and extensibility of short hamstrings. Arch Phys Med Rehabil. 1999;80(4):407-14.

25. Magnusson SP. Passive properties of human skeletal muscle during stretch maneuvers. Scand J Med Sci Sports. 1998;8(2):65-77.

26. Magnusson SP, Aagard P, Simonsen E, Bojsen-Møller F. A biomechanical evaluation of cyclic and static stretch in human skeletal muscle. Int J Sports Med. 1998;19(5):310-6. 
27. Mcnair PJ, Dombroski EW, Hewson DJ, Stanley SN. Stretching at the ankle joint: viscoelastic responses to holds and continuous passive motion. Med Sci Sports Exerc. 2001;33(3):354-8.

28. Morse CI, Degens H, Seynnes OR, Maganaris CN, Jones DA. The acute effect of stretching on the passive stiffness of the human gastrocnemius muscle tendon unit. J Physiol. 2008;586(1):97-106.

29. Karloh M, Santos RP, Kraeski MH, Matias TS, Frutuoso AS. Alongamento estático versus conceito Mulligan efeitos crônicos no treino de flexibilidade em ginastas. Rev Bras Cineantrop Desemp Hum. 2010;12(3):202-8.

Recebido: 19/03/2009

Received: 03/19/2009

Aprovado: 29/06/2010

Approved: 06/29/2010 